\title{
Renewal of extinguished responding in a second context
}

\author{
MARK E. BOUTON and SEAN T. RICKER \\ University of Vermont, Burlington, Vermont
}

\begin{abstract}
In three experiments with rats, we demonstrated that a conditioned response that is learned and extinguished in one context (Context A) can be renewed when the conditioned stimulus (CS) is tested in a second context (Context B). In Experiments 1 and 3, the effect was observed in conditioned suppression; in Experiment 2, it was produced in appetitive conditioning. The result occurs when Contexts A and B are equally familiar, equally associated with reinforcement, or equally associated with both reinforcement and nonreinforcement. The results extend the range of conditions known to produce the renewal effect, and they are consistent with the view that retrieval of extinction depends more on the context than does retrieval of conditioning.
\end{abstract}

The results of a number of experiments suggest that extinction performance depends on the context in which the conditioned stimulus (CS) is presented. For example, if the CS is paired with an unconditioned stimulus (US) in one context, and then is presented alone and extinguished in another context, a return to the original context can "renew" extinguished responding to the CS (e.g., Bouton \& Bolles, 1979; Bouton \& King, 1983; Bouton \& Peck, 1989; Lovibond, Preston, \& Mackintosh, 1984). Renewed responding also occurs if the CS is tested in a third context after extinction in a second context (e.g., Bouton \& Bolles, 1979; Bouton \& Brooks, 1993; see also Bouton \& Swartzentruber, 1986). Extinction performance is thus relatively specific to the context in which it is learned. In contrast, when the context is switched after conditioning, there is often no detectable loss in performance to the CS (e.g., Bouton \& King, 1983; Bouton \& Peck, 1989; Bouton \& Swartzentruber, 1986; Hall \& Honey, 1989; Kaye \& Mackintosh, 1990; Kaye, Preston, Szabo, Druiff, \& Mackintosh, 1987). It appears that extinction is more sensitive to the effects of a context switch than is conditioning.

The role of context in extinction is consistent with the view that extinction involves memory retrieval: Information from both conditioning and extinction is retained, and performance depends on which is retrieved by the current context (Bouton, 1991, 1993). One version of this idea is represented in the model depicted in Figure 1 (Bouton, 1993, 1994; Bouton \& Nelson, 1994). During conditioning, the CS is associated with the US. During extinction, instead of unlearning the original association, a new inhibitory association is formed between the CS and US. Activation of the inhibitory asso-

This research was supported by Grant IBN 92-09454 from the National Science Foundation. Correspondence should be sent to M. E. Bouton, Department of Psychology, University of Vermont, Burlington, VT 05405. ciation prevents conditioned performance. Although retrieval of both associations may be controlled to some extent by context, for reasons mentioned previously, the model assumes that context is especially important in controlling the inhibitory association (Bouton, 1993). As can be seen in the figure, the inhibitory association is modulated by a "control element" that works like an AND gate (e.g., Estes, 1976) so that activation of the inhibitory association depends on input from both the CS and the context (Bouton, 1993). Thus, when the context is changed after extinction, activation of the inhibitory link is reduced, and excitatory responding (renewal) is observed. If temporal cues are coded as part of the context, the model also produces spontaneous recovery (Bouton, 1993); a retention interval after extinction moves the subject out of the extinction context. The model addresses the effects of time and context on extinction performance, and it has been useful for accounting for some novel effects of context on conditioned inhibition (Bouton \& Nelson, 1994).

A clear prediction of the model in Figure 1 is that renewed responding should occur whenever an extinguished CS is tested in a context that differs from the extinction context. Renewal is predicted in a second context (e.g., Context B) even if both conditioning and extinction have been conducted in the same context (e.g., Context A). This particular renewal effect, hereafter called "AAB renewal," has received relatively little attention. As described, renewal has been demonstrated when conditioning, extinction, and testing have occurred in Contexts A, B, and A, or A, B, and C. But relatively little effort has been directed toward demonstrating renewal in an $A A B$ design. Some operant conditioning results may be consistent with the possibility that AAB renewal occurs (see Bouton \& Swartzentruber, 1991, p. 131), and recent conditioned inhibition experiments also suggest that a CS with both excitatory and inhibitory associations may lose its inhibition when it is 


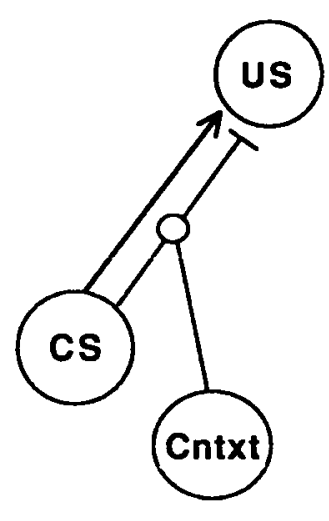

Figure 1. Hypothetical memory structure for extinction. Arrow indicates excitatory association between $C S$ and US learned during conditioning; blocked line indicates inhibitory association learned during extinction. Inhibitory association requires input from both the $C S$ and the context (Cntxt) for activation. From "Context, Ambiguity, and Classical Conditioning," by M. E. Bouton, 1994, Current Directions in Pyychological Science, 3, pp. 49-53. Copyright 1994 by Cambridge University Press. Adapted by permission. See akso Bouton and Nelson (1994).

tested in a second context (Bouton \& Nelson, 1994). However, two previous experiments from this laboratory did not produce $\mathrm{AAB}$ renewal after extinction (Bouton \& King, 1983, Experiment 4; Bouton \& Swartzentruber, 1989, Experiment 2). Because those experiments were partly designed to address other questions, it is possible that $\mathrm{AAB}$ renewal was not tested under optimal conditions. Bouton and King ran extra tests with a second CS between extinction and renewal testing, and they did not equate the rats' overall exposure to the two contexts. And because of an equipment failure, Bouton and Swartzentruber (1989) had only 4 subjects in a control group that could have shown $\mathrm{AAB}$ renewal. Thus, the data neither confirm nor exclude the possibility of $A A B$ renewal after extinction in Pavlovian learning.

The purpose of the present experiments, then, was to examine AAB renewal in more detail. To increase statistical power, we used a relatively large number of subjects and within-subjects test procedures. The results suggest, for the first time, that AAB renewal does occur after extinction in both aversive and appetitive conditioning.

\section{EXPERIMENT 1}

In the first experiment, we used the conditioned suppression method. Sixteen rats were first trained to leverpress for food reward in two contexts (A and B). A houselight-off CS (L) was then paired with a footshock US in Context A and was extinguished in the same context. There were two sessions per day; one session was conducted in Context A (with the Pavlovian events superimposed on baseline responding), and the other session was spent leverpressing in Context $\mathrm{B}$. The procedure thus equated exposure and baseline practice in the two contexts. After extinction, all the animals received four tests of the CS in Context A and four tests in Context $B$ (sequence counterbalanced). If extinguished responding is renewed in a second context, there should be more suppression in Context B than in Context A.

\section{Method}

\section{Subjects}

The subjects were 16 female Wistar rats bred at the University of Vermont. They were approximately 90 days old at the start of the experiment and were individually housed in suspended stainless steel cages in a room maintained on an 18:6-h light:dark cycle. The experiment was conducted on consecutive days during the light portion of the cycle. The rats were food deprived to $80 \%$ of their free-feeding weights and were maintained at that level throughout the experiment.

\section{Apparatus}

We used two sets of four Skinner boxes that were located in two separate rooms. These provided two discriminably different contexts. Each box in one set measured $26 \times 25 \times 19 \mathrm{~cm}$. The front, back, and one side wall were made of aluminum, and the ceiling and other side wall were made of clear acrylic plastic. The floor consisted of tubular steel bars, $16 \mathrm{~mm}$ in diameter, spaced $3.2 \mathrm{~cm}$ center to center, and mounted perpendicular to the front wall. On the front wall of each box, $1 \mathrm{~cm}$ above the floor and centered $11.5 \mathrm{~cm}$ from the right wall, was a recessed $4 \times 4 \mathrm{~cm}$ food cup. A $2.5 \times 2.5 \mathrm{~cm}$ lever protruded from the front wall $5 \mathrm{~cm}$ above the floor and $1 \mathrm{~cm}$ to the right of the food cup. To provide a distinctive scent cue, a dish containing $10 \mathrm{ml}$ of distilled white vinegar $(\mathrm{H}$. J. Heinz Co., Pittsburgh, PA) was placed in the sound-attenuation chamber in which each box was housed. The subjects were placed in the boxes through a door in the right wall.

Each box in the second set measured $24 \times 22 \times 18 \mathrm{~cm}$. The front and back walls were aluminum, and the ceiling and side walls were clear acrylic plastic with vertical black stripes, $2 \mathrm{~cm}$ wide and $2.5 \mathrm{~cm}$ apart. The floor consisted of stainless steel bars, $3 \mathrm{~mm}$ in diameter, spaced $1.5 \mathrm{~cm}$ from center to center, and mounted parallel to the front wall. On the front wall of each box, $1 \mathrm{~cm}$ above the floor and centered $3.5 \mathrm{~cm}$ from the right wall, was a recessed $4 \times 4 \mathrm{~cm}$ food cup. A $4 \times 1 \mathrm{~cm}$ lever protruded from the front wall, $5 \mathrm{~cm}$ above the floor and $6 \mathrm{~cm}$ to the left of the food cup. A dish containing $1 \mathrm{~g}$ of Vick's Vaporub (Richardson-Vicks, Inc., Shelton, CT) was placed in each sound-attenuation chamber to provide a distinctive scent cue. The subjects were placed into the boxes through the ceiling.

In both sets of boxes, illumination was provided by two $7.5-\mathrm{W}$ white incandescent bulbs mounted on the ceilings of the soundattenuation chambers, $25 \mathrm{~cm}$ above the floor. The conditioned stimulus consisted of the 60 -sec offset of these bulbs. This lightoff cue (L) produced complete darkness. The unconditioned stimulus was a $0.6-\mathrm{mA}, 0.5$-sec scrambled footshock, produced by Grason-Stadler shock sources. Operant responding was reinforced with 45-mg food pellets (traditional formula, P. J. Noyes, Lancaster, $\mathrm{NH}$ ). The apparatus was controlled by computer equipment located in an adjacent room.

\section{Procedure}

Pretraining. The subjects first received $30 \mathrm{~min}$ of exposure to the boxes used as Contexts A and B. Boxes comprising contexts were counterbalanced in each group. Food cups were baited with four pellets each prior to the start of each exposure session. In addition, levers were baited with crushed food pellets.

Baseline training. On the following day, the rats received two 60 -min sessions of shaping in Context A. Food cups and levers were baited as before. The subjects were rewarded with food pellets for approaching and pressing the lever located at the front of 
the box. After the operant was established, they were rewarded with a food pellet for each of the first 30 responses. Then they progressed to leaner schedules of reinforcement until they were performing on a $90-\mathrm{sec}$ variable interval (VI-90) schedule. This procedure was repeated the next day in Context B. For the next 3 days, the subjects received one $90-$ min session per day of VI-90 training in each of the two contexts. Throughout the rest of the experiment, they were rewarded for leverpressing in both contexts on a VI-90 schedule of reinforcement.

Conditioning. Following baseline training, the subjects received 3 days of conditioning. On each day, they received one session in each context. Sessions in Context A consisted of four presentations of $\mathrm{L}$, terminating in the onset of the footshock. The mean intertrial interval (ITI) was $22 \mathrm{~min}$. Sessions in Context B consisted of VI-90 reinforcement in the apparatus; no Pavlovian stimuli were presented during these sessions. One squad of rats received sessions in the order AB BA, and so on, and the other squad received them in the reverse order. This pattern was continued until testing. All sessions were $90 \mathrm{~min}$ in length.

Extinction. The rats then received 4 days of extinction. On each day, they received one 90 -min session in Context $A$ and one in Context B. Sessions in Context A consisted of eight presentations of $\mathrm{L}$, with a mean ITI of $10 \mathrm{~min}$. Sessions in Context B consisted of VI-90 reinforcement in the apparatus. No footshocks were presented during this phase.

Testing. Following extinction, the rats received 2 days of testing. On each day, they received one 60 -min session, during which $\mathrm{L}$ was presented four times with a mean ITI of $14 \mathrm{~min}$. One squad received testing in Context $B$ first (on Day 1), and then in Context A (on Day 2). The order was reversed for the other squad. Assignment to the new squads was arranged so that half of each squad came from each of the original squads. No footshocks were presented on these days.

Data analysis. Conditioned responding was indexed by using suppression ratios of the form $x /(x+y)$, where $x$ represents the number of barpresses made during the 60-sec CS, and $y$ represents the number of barpresses made during the $60 \mathrm{sec}$ immediately preceding the $\mathrm{CS}$ (the pre-CS period). A suppression ratio of 0 indicates complete barpress suppression during the $C S$, and a suppression ratio of 0.5 indicates no suppression during the $C S$. Suppression ratios were analyzed with an analysis of variance (ANOVA). A priori hypotheses were also evaluated by using the standard methods described by Howell (1992, pp. 341-355 and pp. 448-470). Barpress rates during the pre-CS period were also analyzed by using ANOVAs identical to those used to analyze suppression ratios. A rejection criterion of $p<.05$ was used throughout.

\section{Results}

Conditioning proceeded uneventfully; the rats showed strong suppression to L by Day 2 . The mean suppression ratio for the last conditioning trial was 0.15 . Extinction proceeded slowly, with the rats still showing slight suppression to L on Day 4. The session mean for Day 4 was 0.45 , which differed significantly from a hypothetical population mean of $0.50[t(15)=-3.76]$. The mean suppression ratio for the last extinction trial (see Figure 2), however, did not differ significantly from 0.50 $[t(15)=-1.19, p>.25]$.

The data of main interest are shown in Figure 2, which shows suppression ratios for all 16 subjects on the last extinction trial and on the test trials in Contexts $A$ and $B$. A context $x$ order $x$ trial ANOVA carried out on the test data revealed a significant main effect of context

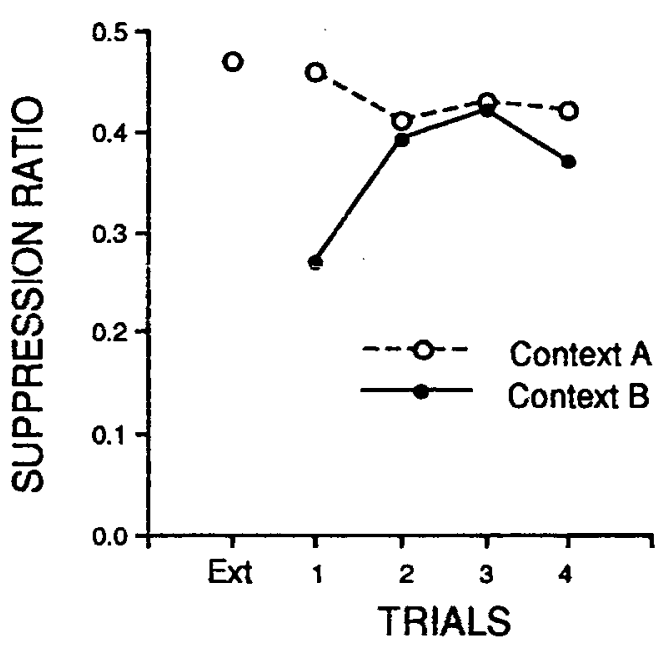

Figure 2. Mean suppression to the light-off CS on the last trial of extinction in Context A (Ext) on each of the test trials conducted in Contexts $A$ and $B$ in Experiment 1.

$[F(1,14)=26.66]$. The subjects suppressed significantly more when tested in Context $\mathrm{B}$. There was also a significant context $\times$ trial interaction $[F(3,42)=3.87]$ and a significant context $\times$ order interaction $[F(1,14)=$ 4.81]. Although the animals tested first in Context B tended to show a stronger context effect than those tested first in Context A, there was significantly more suppression in Context B than in Context A on the first test trial with either order $[F(1,51)>7.63]$. All other effects were nonsignificant $(F \mathrm{~s}<2.17)$.

There were no differences in leverpressing during the pre-CS period of the test sessions, when the subjects responded an average of 37.6 in Context $A$ and 34.6 in Context B. A similar ANOVA, carried out on the pre-CS data for testing, revealed no significant effects $(F \mathrm{~s}<$ 2.80 ).

\section{Discussion}

After extinction, there was stronger suppression in a second context than in the context in which conditioning and extinction had both occurred. These results clearly suggest that $\mathrm{AAB}$ renewal can occur in the conditioned suppression preparation. With the present method, extinction performance was more sensitive to a change of context than was conditioning performance.

\section{EXPERIMENT 2}

In studies of conditioned suppression, it is common to observe some unconditional suppression of baseline responding when the CS is first presented at the outset of conditioning. This suppression presumably occurs because the rat makes an orienting response that is incompatible with leverpress responding. The response typically habituates within one or two trials (e.g., Leaton, 1974). But since it is theoretically possible that habitu- 
ation is context specific (Wagner, 1976, 1981), dishabituation of the orienting response could have occurred with the context switch and thus contributed to the renewal effect observed in Experiment 1.

One very serious problem for this interpretation is that there is virtually no evidence that dishabituation occurs when a habituated stimulus is presented in a second, equally familiar context (e.g., Churchill, Remington, \& Siddle, 1987; Hall \& Channell, 1985; Marlin \& Miller, 1981). In fact, in the barpress suppression paradigm, habituated unconditioned suppression appears to transfer perfectly between contexts (e.g., Baker \& Mercier, 1982; Hall \& Honey, 1989; Leaton, 1974; see also Bouton \& Brooks, 1993). In an unpublished experiment that shared all the details of Experiment 1 except that the rats were never shocked, we likewise found no evidence of a dishabituation of unconditioned suppression to $L$ when $\mathrm{L}$ was tested in Context $\mathrm{B}$ after habituation in Context $\mathrm{A}$. Nevertheless, in Experiment 2, we pursued the possibility even further by extending the generality of the results of Experiment 1 from aversive to appetitive conditioning. Here the CS was paired with a food pellet US, and conditioning was indexed by the number of times the rat entered the food cup during the CS (e.g., Bouton \& Nelson, 1994; Brooks \& Bouton, 1993; Hall \& Channell, 1985; Hall \& Honey, 1990; Kaye \& Mackintosh, 1990). In this preparation, dishabituation of an orienting response with a context switch would presumably reduce, rather than enhance, the conditioned magazine-entry response. Previous work in our laboratory suggests that the conditioned magazine-entry response is not changed when the context is switched after conditioning (e.g., Bouton \& Nelson, 1994).

\section{Method}

\section{Subjects}

The subjects were 3 male and 19 female Wistar rats. They were approximately 100 days old at the start of the experiment, and procedures for housing and maintenance were the same as those in Experiment 1.

\section{Apparatus}

We used two new sets of four Skinner boxes, which were located in sound-attenuation chambers in two separate rooms. Each box in either set was made of clear acrylic plastic and measured $23 \times$ $13 \times 11 \mathrm{~cm}$. The outsides of the rear and side walls were covered with black construction paper, and the front wall and ceiling were left transparent. A recessed stainless steel food cup was positioned $3 \mathrm{~cm}$ above the floor on the right wall of each box; access was available through a 6 -cm-square opening. Illumination was provided by two $7.5-\mathrm{W}$ white incandescent bulbs, mounted $25 \mathrm{~cm}$ above the floor on the ceiling of each sound-attenuation chamber. The subjects were placed in the boxes through the ceiling.

Each box in one set had floors consisting of $3-\mathrm{mm}$ bars mounted parallel to the side wall. The bars were staggered in such a way that the odd-numbered bars were mounted $6 \mathrm{~mm}$ above the evennumbered bars; the distance between consecutive bars was $1.6 \mathrm{~cm}$. The three black (covered) walls were lined with horizontal white stripes, $1 \mathrm{~cm}$ wide and spaced $1 \mathrm{~cm}$ apart. A dish containing $10 \mathrm{ml}$ of $4 \%$ coconut extract solution (McCormick \& Co., Inc., Hunt Valley, MD) was placed within each sound-attenuation chamber to provide a distinctive scent cue. The second set of boxes had floors consisting of 3-mm bars, spaced $1.8 \mathrm{~cm}$ apart and mounted diagonally with respect to the chamber walls. The three covered walls were black. A dish of $2 \%$ McCormick anise extract solution was positioned within each chamber to provide a distinctive scent cue. These sets of boxes provided the two contexts used in the experiment.

Two conditioned stimuli were used. One was a 30 -sec presentation of a $3000-\mathrm{Hz}$ tone ( $80 \mathrm{~dB}$ re $20 \mu \mathrm{N} / \mathrm{M}^{2}[\mathrm{~A}]$ ), which was provided by a generator wired to identical speakers mounted in each chamber $25 \mathrm{~cm}$ above the box floor. Background noise level was $65 \mathrm{~dB}$. The other CS was a $30-\mathrm{sec}$ presentation of a GE 313 (General Electric Co., Cleveland, $\mathrm{OH}$ ) keylight that was mounted on the outer side of the rear wall, $13 \mathrm{~cm}$ from the floor and $4.5 \mathrm{~cm}$ from the right wall of each box. A 3-cm-diam hole in the construction paper covering the rear wall allowed the keylight to be visible from the inside of each box. The US consisted of two 45-mg Noyes food pellets (traditional formula) delivered $0.2 \mathrm{sec}$ apart. Magazine entries were detected by photocells mounted within the food cups, just behind the plane of the wall of the Skinner boxes. The apparatus was controlled by computer equipment located in an adjacent room.

\section{Procedure}

Pretraining. The subjects first received $30 \mathrm{~min}$ of exposure to Contexts A and B. (Contexts were counterbalanced as in the previous experiment.) Food cups were baited with 4 food pellets each, prior to the start of each exposure session. On the following day, the subjects received one 30 -min session of magazine training in both contexts. Food cups were again baited with 4 pellets each. During each session, the subjects were trained to approach and eat from the food cup when the feeder mechanism was activated. Approximately 20 pellets were delivered during each session.

Conditioning. Following magazine training, the subjects received 5 days of conditioning. On each day, they received one session in Context A and one in Context B. Sessions in Context A consisted of 16 presentations of the tone ( $T$ ) followed by the food US, with a mean ITI of $270 \mathrm{sec}$. Sessions in Context B consisted of 16 presentations of the keylight $(\mathrm{K})$ followed by the food US, with a mean ITI of $270 \mathrm{sec}$. (This procedure ensured that the rats learned that the food cup was equally effective in Contexts $A$ and B.) One squad of 14 rats received sessions in the order $A B B A$, and so on, while a second squad of 8 received the reverse order. The pattern was continued until testing. All sessions were $88 \mathrm{~min}$ long.

Extinction. Following conditioning, the subjects received 3 days of extinction. On each day they received one session in Context $\mathrm{A}$, in which $\mathrm{T}$ was presented 24 times alone. The first ITI was $11 \mathrm{~min}$ in duration; thereafter they were $190 \mathrm{sec}$. In addition, on each day the subjects received one session of exposure to Context $B$ (no stimuli were presented). No food was presented during this phase. All sessions were $108 \mathrm{~min}$ long.

Testing. Following extinction, the rats received 2 days of testing. On each day they received one 53-min session, in which $\mathrm{T}$ was presented eight times with a mean ITI of $333 \mathrm{sec}$. No food was delivered. Half of the subjects from each of the previous squads were tested in Context B on Day 1 and in Context A on Day 2. For the other half, this order was reversed.

Data analysis. Conditioned responding was measured as the number of magazine entries made during the 30-sec CS. The CS scores were analyzed by ANOVAs similar to those used in Experiment 1 . Responding during the $30-\mathrm{sec}$ pre-CS period was also analyzed by using an ANOVA identical to that used to analyze the CS scores.

\section{Results}

Conditioning and extinction proceeded uneventfully. Mean CS scores for the last conditioning and extinction trials with $T$ were 10.54 and 0.36 , respectively. 
The data of primary interest are presented in Figure 3, which shows CS scores for the last two-trial block of extinction and the two-trial blocks for testing in Contexts $A$ and $B$. The test data were subjected to a context $x$ order $\times$ block ANOVA. The analysis revealed a significant main effect of context $[F(1,20)=6.58]$; the subjects responded significantly more when tested in Context $\mathrm{B}$. The block effect was also reliable $[F(3,60)=$ $8.22]$; responding tended to decrease over trials, regardless of the test context. No other effects approached significance $(F \mathrm{~s}<2.56)$. A separate ANOVA revealed that test performance on the first two-trial block in Context A was higher than that on the last two-trial block of extinction $[F(20)=15.18]$, suggesting that some spontaneous recovery was also evident during testing. The main result, however, was that there was once again significantly more responding when the CS was tested in Context $\mathrm{B}$ after conditioning and extinction in Context A.

There were no problematic differences in magazine entries during the pre-CS period, which averaged 0.56 and 0.77 for testing in Contexts A and B, respectively. An identical ANOVA carried out on prescores revealed only a significant effect of block $[F(3,60)=4.34]$. Responding during the pre-CS period tended to decrease over trials, but it did not differ between contexts.

\section{Discussion}

The results of this experiment extend the results of Experiment 1 to an appetitive conditioning situation. As in Experiment 1, extinction performance was again reduced when the context was switched after extinction. Because an orienting response to the CS would be likely to interfere with, rather than facilitate, the conditioned magazine-entry response used here, it is unlikely that renewal resulted from dishabituation of an orienting response. This conclusion is consistent with the wealth of

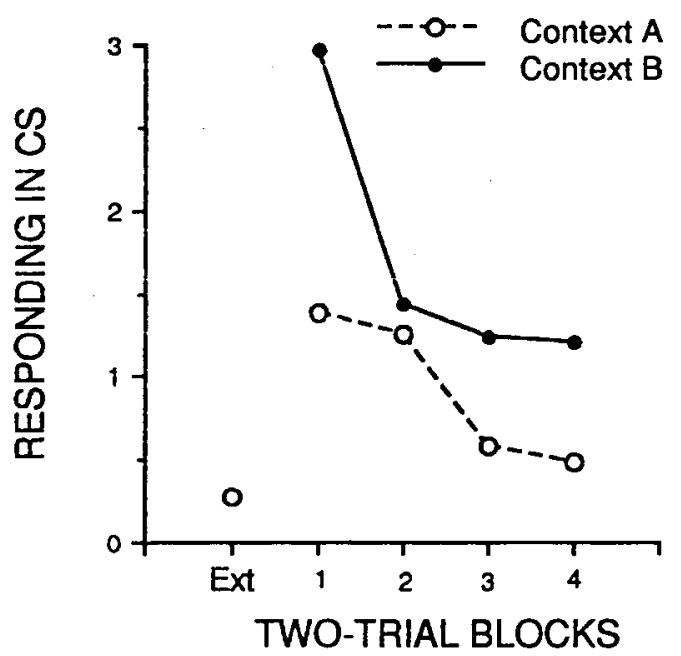

Figure 3. Mean responding during the tone CS (CS scores) during the last two-trial block of extinction in Context $A$ (Ext) and on the twotrial blocks of testing in Contexts $A$ and $B$ in Experiment 2. data suggesting that dishabituation does not occur with a context switch (e.g., Baker \& Mercier, 1982; Bouton \& Brooks, 1993; Churchill et al., 1987; Hall \& Channell, 1985; Hall \& Honey, 1989; Leaton, 1974; Marlin \& Miller, 1981). The results of Experiment 2, coupled with the general lack of evidence that context switches cause dishabituation, very strongly suggest that $A A B$ renewal is not the result of dishabituation occurring with the context switch.

\section{EXPERIMENT 3}

In the third experiment, we returned to the conditioned suppression preparation and further analyzed the renewal effect observed in Experiment 1. In Experiment 3, we asked whether $\mathrm{AAB}$ renewal still occurs when $A$ and $B$ are equated more completely on their possible excitatory and inhibitory associations with the US. There were two groups. The first group (Group L/-) received the treatment investigated in Experiment 1: The rats received conditioning and extinction of $\mathrm{L}$ in Context $A$ with intermixed sessions of simple exposure (baseline responding) in Context $\mathrm{B}$. The second group (Group $L / N$ ) received the same sessions in Context $A$, but the sessions in Context $\mathrm{B}$ involved conditioning and extinction of an intermittent white noise CS (N) in a manner that paralleled the treatment of $L$. That is, for every session of conditioning or extinction of $L$ in Context $A$, there was a comparable session of conditioning or extinction of $\mathrm{N}$ in Context $\mathrm{B}$.

This method of equating the contexts on associative strength was first introduced by Lovibond et al. (1984), who found renewed responding to the CS when it was conditioned in Context A, extinguished in Context B, and finally tested in Context A. However, no renewal was observed in a separate experiment in which a group received parallel conditioning and extinction with two CSs in the two contexts. The results suggested that renewal may depend on differential treatment of the two contexts. However, recent research in our laboratory suggests that inhibition to a CS may be reduced by a context switch even when the contexts receive parallel treatments (Bouton \& Nelson, 1994). It was therefore worth asking whether the parallel treatment would reduce renewal by comparing performance in Groups $\mathrm{L} / \mathrm{-}$ and $\mathrm{L} / \mathrm{N}$ in the $\mathrm{AAB}$ renewal design. $\mathrm{L}$ remained the target $\mathrm{CS}$, because complete darkness should be perceived as the same stimulus in the two contexts.

\section{Method}

\section{Subjects}

The subjects were 3 male and 13 female Wistar rats from the same stock that was used in the previous experiments; housing and maintenance were also the same. All except 1 male had previously served in Experiment 2. The rats were approximately 140 days old at the start of the experiment.

\section{Apparatus}

The apparatus was the same as that used in Experiment 1 (which differed from that used in Experiment 2). A second CS was added. 
The new stimulus was a 60 -sec presentation of an intermittent white noise ( 6 pulses $/ \mathrm{sec}, 65 \mathrm{~dB}$ re $\left.20 \mu \mathrm{N} / \mathrm{M}^{2}[\mathrm{~A}]\right)$. This stimulus was provided by a generator wired to identical speakers that were mounted $25 \mathrm{~cm}$ above the floor within the sound-attenuation chambers.

\section{Procedure}

Baseline training. Baseline training was the same as that in Experiment 1, with the exception that, on Day 1, the rats received two 60 -min sessions of barpress shaping-one in Context $\mathrm{A}$ and one in Context B. For the next 4 days, the subjects received one 90 -min session per day VI-90 training in each of the two contexts. Throughout the rest of the experiment, they were rewarded for leverpressing in both contexts on a VI-90 schedule of reinforcement.

Conditioning. Following baseline training, the rats received 3 days of conditioning. On each day, they received one session in Context $\mathrm{A}$ and one in Context B. For both groups, sessions in Context $A$ contained four presentations of $L$, terminating at the onset of the footshock. The mean ITI was $22 \mathrm{~min}$. For Group L/-, sessions in Context B consisted of VI-90 training in the apparatus; no Pavlovian stimuli were presented. For Group L/N, sessions in Context $\mathrm{B}$ consisted of four parallel presentations of $\mathrm{N}$, terminating in footshock. All the subjects received sessions in the order A B. All sessions were $90 \mathrm{~min}$ in length.

Extinction. Following conditioning, the rats received 4 days of extinction. On each day, they again received one session in Context $\mathrm{A}$ and one in Context B. For both groups, sessions in Context A consisted of eight presentations of $L$ with a mean ITI of 10 min. For Group L/-, sessions in Context B again consisted of VI-90 training in the apparatus. For Group L/N, sessions in Context $\mathrm{B}$ consisted of eight presentations of $\mathrm{N}$ with a mean ITI of $10 \mathrm{~min}$. No footshocks were presented during this phase. Each session was again $90 \mathrm{~min}$ in length.

Testing. Following extinction, the subjects were given 2 days of testing. Prior to testing, the groups were divided into two squads each. On each day, each squad received one 60 -min session, during which $\mathrm{L}$ was presented four times with a mean ITI of $15 \mathrm{~min}$. One squad in each group received testing in Context B on Day 1, and then in Context A on Day 2. For the other squad, the order was reversed. No footshocks were presented.

\section{Results}

Conditioning and extinction proceeded uneventfully. The mean suppression ratios for the last conditioning trial were 0.14 for Group $\mathrm{L} / \mathrm{-}$ and 0.22 for Group $\mathrm{L} / \mathrm{N}$.

The data of main interest are presented in Figure 4, which shows suppression ratios for Groups $\mathrm{L} /-$ and $\mathrm{L} / \mathrm{N}$ for the last extinction trial with $\mathrm{L}$, and the four tests of $\mathrm{L}$ in Contexts $\mathrm{A}$ and $\mathrm{B}$. The test data were subjected to a group $\times$ context $\times$ test order $\times$ trial ANOVA, which revealed a significant main effect of context $[F(1,12)=$ 8.21]; overall, there was more suppression in Context B than in Context $\mathrm{A}$. The trial effect was also significant $[F(3,36)=4.50]$. However, neither the group effect nor any interaction involving the group factor approached significance $(F \mathrm{~s}<1)$. Simple effects tests on the first trial reliably indicated more suppression in Context B than in Context $\mathrm{A}$ in each of the groups taken individually $[F \mathrm{~s}(1,47)>5.74]$. No other effects from the ANOVA approached significance $(F \mathrm{~s}<2.34)$. It is worth noting that on the first test trial in Context A, overall suppression was significantly lower than a hypothetical population mean of $0.50[t(15)=-2.75]$,

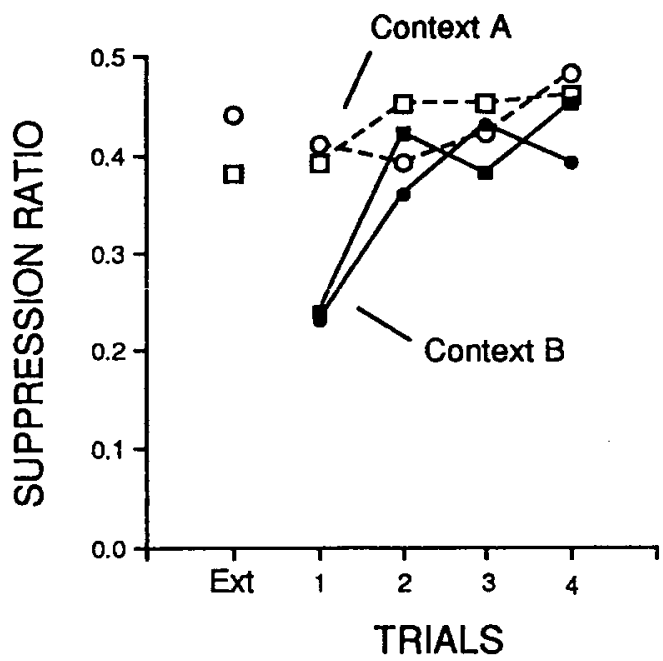

Figure 4. Mean suppression to the light-off CS on the last trial of extinction in Context $A$ (Ext) and on each test trial in Contexts $A$ and B in Experiment 3 (circles $=$ Group $L /-$; squares $=$ Group $L / N$ ).

suggesting that extinction was not quite complete at the outset of testing.

There were no differences in leverpressing during the pre-CS period, when Group L/ - averaged 36.0 and 40.9 responses in Contexts $A$ and $B$, respectively, and Group $L / N$ averaged 39.9 and 41.3. An identical ANOVA carried out on the pre-CS data for testing revealed no significant main effects or interactions $(F \mathrm{~s}<1.05)$.

\section{Discussion}

The results of this experiment replicate and extend the $A A B$ renewal effect observed in Experiment 1. Importantly, the effect appeared to be of comparable size whether or not the context in which renewal was observed (Context B) had received a parallel treatment with a second CS. The observation of renewal in Group $\mathrm{L} / \mathrm{N}$ suggests that the effect is not due to differential association of Contexts A and B with the US. Instead, the renewal effect observed here, like that observed in other designs, may depend on the contexts retrieving $\mathrm{L}$-shock and L-no-shock relations (see Bouton, 1991, 1993, for reviews). The fact that performance recovers when the context is switched following conditioning and extinction suggests that the context is especially important in retrieving the $\mathrm{L}-$-no-shock relation.

\section{GENERAL DISCUSSION}

In each of the present experiments, extinguished responding was renewed when the CS was tested in a second context (Context B) after both conditioning and extinction had been conducted in the same context (Context A). AAB renewal was found in both aversive (Experiments 1 and 3) and appetitive (Experiment 2) conditioning preparations. It was also found regardless of whether the second context was merely as familiar as 
the first context (Experiment 1), was equally associated with the conditioning of a CS (Experiment 2), or was equally associated with both the conditioning and extinction of a CS (Experiment 3 ). The range of conditions in which $\mathrm{AAB}$ renewal was observed here suggests that the effect may have some generality.

Renewal in a second context appears to require that the context switch disrupts extinction more than conditioning; if conditioning were as easy to disrupt as extinction, there would be no basis for conditioned responding in a new context. As mentioned above, this consideration suggests that the hypothetical inhibitory CS-US association learned in extinction is more context dependent than is the excitatory association learned during conditioning. Further, the fact that renewal occurred even when Contexts A and B were equally associated with reinforcement and nonreinforcement (Experiment 3) suggests that the result is not controlled by simple contextUS associations. Instead, the present data suggest that the context must provide a fairly specific cue for the extinction of a particular CS. The results are consistent, then, with the retrieval model of extinction that is presented in Figure 1 (Bouton, 1994; Bouton \& Nelson, 1994).

It may be noted that $\mathrm{AAB}$ renewal was most evident on the first test trial. This feature of renewal is consistent with other postextinction recovery effects, including spontaneous recovery and reinstatement, wherein extinguished performance recovers after the passage of time (e.g., Brooks \& Bouton, 1993; Robbins, 1990) or after presentation of the US (e.g., Bouton \& King, 1983; Rescorla \& Heth, 1975), respectively. One interpretation of this feature of postextinction recovery is that the first test trial itself has the ability to retrieve the representation of extinction (see Brooks \& Bouton, 1993). In the model presented in Figure 1, previous extinction trials may comprise part of the context in which the CS was extinguished.

The present findings may have practical implications. In principle, the results suggest that the effects of therapeutic treatments designed to extinguish maladaptive behavior may be disrupted by a change of context after extinction. However, it is possible that AAB renewal depends on particular test procedures. For example, excitation can be attenuated with a context switch under some conditions (e.g., Hall \& Honey, 1989, 1990); it seems probable that $\mathrm{AAB}$ renewal would also be weaker under them. In addition, it is not known whether $A A B$ renewal would survive more extended extinction training. These observations do not undermine the conclusion that extinction performance is more sensitive to context change than is conditioning performance; instead, they indicate that we have yet to establish the boundary conditions of the $A A B$ renewal effect. It is worth noting that the more extensively studied $A B A$ renewal effect does not appear to be eliminated by fairly extensive extinction training (see Bouton \& Swartzentruber, 1991, for a review).

Kaye and Mackintosh (1990) found that conditioned responding to a CS that has never been extinguished can sometimes increase when the CS is switched to a new context. At least two considerations suggest that $A A B$ renewal is not merely a new example of this "supertransfer" effect. First, Kaye and Mackintosh observed supertransfer in conditioned suppression, but not in an appetitive magazine-entry procedure similar to the one used in the present Experiment 2. AAB renewal thus seems less restricted to conditioned suppression. Second, supertransfer may be further restricted to conditioned suppression procedures in which the two contexts are both associated with conditioning. For example, in experiments by Swartzentruber and Bouton (1986), a context switch did not produce supertransfer even though responding was not on a response floor. In those experiments, the switch was to a context that was equally familiar, but had not been associated with conditioning of a second CS. In a related experiment, Swartzentruber (1986, Experiment 3) observed supertransfer when the CS was switched to a context that had been associated with the parallel conditioning of a second CS. The latter method was the one used by Kaye and Mackintosh. AAB renewal clearly does not depend on this method; use of the parallel conditioning procedure also had no discernible effect on renewal in Experiment 3. Although it is possible that $\mathrm{AAB}$ renewal and supertransfer are connected at a theoretical level, the evidence suggests that the present results are not merely a new example of supertransfer.

In the main, the present results extend the range of conditions known to produce the renewal effect. The results of experiments on renewal with the ABA, the ABC, and now the $A A B$ design are all consistent with the view that extinction performance is more context specific than is conditioning performance.

\section{REFERENCES}

Baker, A. G., \& Mercier, P. (1982). Prior experience with the conditioning events: Evidence for a rich cognitive representation. In A. R. Wagner, R. Herrnstein, \& M. Commons (Eds.), Quantitative analysis of behavior: Acquisition processes (pp. 117-144). Cambridge, MA: Ballinger.

Bouton, M. E. (1991). Context and retrieval in extinction and in other examples of interference in simple associative learning. In L. Dachowski \& C. F. Flaherty (Eds.), Current topics in animal learning: Brain, emotion, and cognition (pp. 25-53). Hillsdale, NJ: Erlbaum.

Bouton, M. E. (1993). Context, time, and memory retrieval in the interference paradigms of Pavlovian learning. Psychological Bulletin, 114, 80-99.

Bouton, M. E. (1994). Context, ambiguity, and classical conditioning. Current Directions in Psychological Science, 3, 49-53.

Bouton, M. E., \& Bolles, R. C. (1979). Contextual control of the extinction of conditioned fear. Learning \& Motivation, 10, 445-466.

Bouton, M. E., \& Brooks, D. C. (1993). Time and context effects on performance in a Pavlovian discrimination reversal. Journal of Experimental Psychology: Animal Behavior Processes, 19, 165-169.

Bouton, M. E., \& KING, D. A. (1983). Contextual control of the extinction of conditioned fear: Tests for the associative value of the context. Journal of Experimental Psychology: Animal Behavior Processes, 9, 248-265.

Bouton, M. E., \& Nelson, J. B. (1994). Context-specificity of target vs. feature inhibition in a feature negative discrimination. Journal of Experimental Psychology: Animal Behavior Processes, 20, 51-65. Bouton, M. E., \& PecK, C. A. (1989). Context effects on condition- 
ing, extinction, and reinstatement in an appetitive conditioning preparation. Animal Learning \& Behavior, 17, 188-198.

Bouton, M. E., \& Swartzentruber, D. (1986). Analysis of the associative and occasion-setting properties of contexts participating in a Pavlovian discrimination. Journal of Experimental Psychology: Animal Behavior Processes, 12, 333-350.

Bouton, M. E., \& SWARTZENTRUBer, D. (1989). Slow reacquisition following extinction: Context, encoding, and retrieval mechanisms. Journal of Experimental Psychology: Animal Behavior Processes, 15, 43-53.

Bouton, M. E., \& Swartzentruber, D. (1991). Sources of relapse after extinction in Pavlovian and instrumental learning. Clinical Psychology Review, 11, 123-140.

Brooks, D. C., \& Bouton, M. E. (1993). A retrieval cue for extinction attenuates spontaneous recovery. Journal of Experimental Psychology: Animal Behavior Processes, 19, 77-89.

Churchill, M., Remington, B., \& Siddle, D. A. T. (1987). The effects of context change on long-term habituation of the orienting response in humans. Quarterly Journal of Experimental Psychology, 39B, 315-338

EsTES, W. K. (1976). Structural aspects of associative models for memory, In C. N. Cofer (Ed.), The structure of human memory. San Francisco: W. H. Freeman.

Hall, G., \& Channell, S. (1985). Differential effects of contextual change on latent inhibition and on the habituation of an orienting response. Journal of Experimental Psychology: Animal Behavior Processes, 11, 470-481.

Hall, G., \& Honey, R. C. (1989). Contextual effects in conditioning, latent inhibition, and habituation: Associative and retrieval functions of contextual cues. Journal of Experimental Psychology: Animal Behavior Processes, 15, 232-241.

Hall, G., \& Honey, R. C. (1990). Context-specific conditioning in the conditioned-emotional-response procedure. Journal of Experimental Psychology: Animal Behavior Processes, 16, 271-278.

Howell, D. C. (1992). Statistical methods for psychology (3rd ed.). Boston: PWS-Kent.

KAYE, H., \& MaCKINTOSH, N. J. (1990). A change of context can enhance performance of an aversive but not of an appetitive conditioned response. Quarterly Journal of Experimental Psychology, 42B, 113-134.
Kaye, H., Preston, G. C., Szabo, L., Druiff, H., \& Mackintosh, N. J. (1987). Context specificity of conditioning and latent inhibition: Evidence for a dissociation of latent inhibition and associative interference. Quarterly Journal of Experimental Psychology, 39B, $127-145$

LEATON, R. N. (1974). Long-term retention of the habituation of lick suppression in rats. Journal of Comparative \& Physiological Psychology, 87, 1157-1164.

Lovibond, P. F., Preston, G. C., \& Mackintosh, N. J. (1984). Context specificity of conditioning, extinction, and latent inhibition. Journal of Experimental Psychology: Animal Behavior Processes, 10, 360-375.

Marlin, N. A., \& Miller, R. R. (1981). Associations to contextual stimuli as a determinant of long-term habituation. Journal of Experimental Psychology: Animal Behavior Processes, 7, 313-333.

Rescorla, R. A., \& HeTH, C. D. (1975). Reinstatement of fear to an extinguished conditioned stimulus. Journal of Experimental Psychology: Animal Behavior Processes, 1, 88-96.

RoBBINS, S. J. (1990). Mechanisms underlying spontaneous recovery in autoshaping. Journal of Experimental Psychology: Animal Behavior Processes, 16, 235-249.

SWARTZENTRUBER, D. (1986). A role for context in Hall-Pearce negative transfer. Unpublished master's thesis, University of Vermont, Burlington.

Swartzentruber, D., \& Bouton, M. E. (1986), Contextual control of negative transfer produced by prior CS-US pairings. Learning \& Motivation, 17, 366-385.

WAGNER, A. R. (1976). Priming in STM: An information-processing mechanism for self-generated or retrieval-generated depression in performance. In T. J. Tighe \& R. N. Leaton (Eds.), Habituation: Perspectives from child development, animal behavior, and neurophysiology (pp. 95-128). Hillsdale, NJ: Erlbaum.

WAGNER, A. R. (1981). SOP: A model of automatic memory processing in animal behavior. In N. E. Spear \& R. R. Miller (Eds.), Information processing in animals: Memory mechanisms (pp. 5-47). Hillsdale, NJ: Erlbaum.

(Manuscript received August 10, 1993; revision accepted for publication February 24, 1994.) 